\title{
Robert C. Fuller, Spiritual but not Religious: Understanding Unchurched America
}

New York, Oxford University Press, 2001, 212 p. (index)

Baptiste Coulmont

\section{(QpenEdition}

\section{Journals}

Édition électronique

URL : http://journals.openedition.org/assr/957

DOI : 10.4000/assr.957

ISSN : $1777-5825$

Éditeur

Éditions de l'EHESS

Édition imprimée

Date de publication : 1 octobre 2003

Pagination : 63-170

ISBN : 2-222-96739-2

ISSN : 0335-5985

\section{Référence électronique}

Baptiste Coulmont, «Robert C. Fuller, Spiritual but not Religious: Understanding Unchurched America », Archives de sciences sociales des religions [En ligne], 124 | octobre - décembre 2003, document 124.61, mis en ligne le 25 octobre 2005, consulté le 24 septembre 2020. URL : http://journals.openedition.org/ assr/957 ; DOI : https://doi.org/10.4000/assr.957 
nationalité et culture et les applique au cas québécois. Elle analyse les solutions possibles pour opérer une jonction entre ces notions, en soulignant trois points : la transformation du nationalisme ethnique en nationalisme civique, les difficultés du projet souverainiste et les atouts d'un fédéralisme asymétrique. La politologue Margaret Moore critique les théories libérales du nationalisme, qu'elle juge inadéquates pour la prise en compte des minorités nationales et pour faire face aux risques de fragmentation des États existants. Les politologues Gilles Bourque et Jules Duchastel retracent l'histoire politique récente du Canada et considèrent que l'État multiculturel canadien est « un fort mauvais substitut de l'État multinational » (p. 159). L'anthropologue Gilles Bibeau interpelle les politologues en leur reprochant de trop peu s'interroger sur la dynamique concrète des contacts entre les ethnies et les anthropologues, écartelés entre la mondialisation et l'étude locale. Il propose un détour par les œuvres littéraires, et en particulier par les récits fondateurs amérindiens, deux littératures nationales qui ont suivi des chemins distincts, et affirme que « les nations sont à l'image des histoires qu'elles se racontent à elles-mêmes » (p. 181). Le politologue Benjamin Barber décrit les mécanismes de la société universelle de consommation, la «culture Mc World», qui «met hors-jeu [...] ses concurrents démocratiques» (p. 211). La sociologue D.H. conclut l'ouvrage par un remarquable article de synthèse sur les diverses approches de la mondialisation, de la citoyenneté et du multiculturalisme, qui permet de situer les contributeurs au colloque, ainsi que les principaux autres penseurs de ces débats, cités ou omis dans le reste de l'ouvrage.

La lecture de ce livre est particulièrement stimulante pour qui s'intéresse aux questions fort actuelles des identités plurielles, d'autant plus que les auteurs s'y interpellent et se répondent, non sans polémique.

Anne-Sophie Lamine.

\subsection{1}

FULLER (Robert C.).

Spiritual but not Religious: Understanding Unchurched America. New York, Oxford University Press, 2001, 212 p. (index).

À la question : «De quelle Église êtes-vous membre? », un grand nombre d'Américains pourraient répondre: «Je suis spirituel, mais pas religieux. " Et il serait intéressant de comprendre ce qu'ils entendent par là. Malgré le sous-titre et certains paragraphes de l'ouvrage, ce n'est pas le propos de l'auteur.
Son but est en effet d'inscrire au centre de la religiosité américaine ce qu'il appelle une tradition de spiritualité « unchurched », séparée des institutions religieuses, ne requérant pas l'adhésion à une Église mais recherchant un langage pour exprimer leurs expériences du sacré. Cependant, jamais l'A. n'étudie les pratiques individuelles ou les «bricolages » personnels : il s'appuie au contraire sur les textes publiés par les fondateurs des divers courants spirituels qu'il analyse. Or, si la floraison de discours est remarquable, celle des pratiques l'est beaucoup moins et nécessite un tout autre travail, ce que reconnaît l'A. : " comprendre les "actes" religieux réels des gens est un jeu de devinettes » (p. 171).

Deux mouvements structurent alors son ouvrage. En premier lieu une démarche historique, où l'A. s'appuie sur ses travaux précédents sur le mesmérisme, la spiritualité de l'inconscient, les médecines alternatives et l'usage des drogues dans l'histoire religieuse américaine. Cette démarche est aussi une démarche de revalorisation de la spiritualité «unchurched»: l'A. construit ainsi l'expression de "great metaphysical awakening " (grand réveil métaphysique, p. 23) sur le modèle du "great religious awakening", moment du XIX ${ }^{\mathrm{e}}$ siècle qui est considéré dans l'histoire religieuse américaine comme le moment fondateur de la religiosité des ÉtatsUnis. L'A. explore alors une foule de mouvements (mesmérisme, swedenborgianisme, transcendentalisme, théosophie, science chrétienne...) en montrant leurs interfécondations et leurs emprunts réciproques.

En deuxième lieu, une démarche sociologique oriente le travail de R.C.F., qui s'appuie alors sur les ouvrages de Wade Clark Roof (Spiritual Marketplace, 1999; A Generation of Seekers, 1993), Robert Wuthnow (After Heaven, 1998) et Robert Bellah (Habits of the Heart, 1985), qui ont décrit les formes émergentes de spiritualité liées à la génération du baby-boom. L'objet sociologique construit par l'A. - l'existence d'un groupe social qui pourrait être décrit au moyen de l'expression « spirituel mais pas religieux » - reste cependant flou : il considère que vingt pour cent des Américains sont non-affiliés à une Église mais doivent être considérés comme religieux «au sens large du terme » (p. 4). Mais il y ajoute aussi ceux qui, affiliés à une église, croient à l'astrologie ou à la réincarnation (p. 99).

L'intérêt de l'ouvrage réside moins dans cette démarche sociologique que dans deux entreprises menées de front. Tout d'abord, comme dans l'ouvrage d'Amanda Porterfield (cf. infra, 124.73), l'A. prend les usages 
psychothérapeutiques de la religion ou de la spiritualité comme objet d'étude : un chapitre est consacré à la «spiritualité psychologique» et à la naissance de la "pastoral psychology».

Le deuxième intérêt provient du but dans lequel l'ouvrage a été rédigé : comme une introduction destinée à un large public (ce que l'A. précise en appendice): les chapitres thématico-historiques (religion méthaphysique; médecines alternatives; relations entre psychologie et spiritualité) et un index bien fourni (plus de 550 entrées) devraient permettre de remplir ce but.

\section{Baptiste Coulmont.}

Les Origines des sœurs de Saint-Joseph au $\mathrm{XVII}^{\mathrm{e}}$ siècle. Histoire oubliée d'une fondation, Saint-Flour-Le Puy (1641-1650-1661). Paris, Cerf, 2000, 608 p. (bibliogr., index, illustr.) (coll. « Petits-Cerf-Histoire »).

M.-L.G. est bien connue par la maîtrise avec laquelle elle a mené ses recherches théologiques et historiques sur Jeanne-Marie Guyon. Elle nous livre aujourd'hui un instrument de travail très élaboré sur une autre facette de la spiritualité française à l'époque classique. Son investigation de première main dans les archives des origines d'une congrégation féminine dépasse l'intérêt d'une monographie approfondie. En même temps, ayant adopté comme démarche d'exposition celle même de la découverte, l'auteure nous fournit une très utile leçon de méthode.

La fondation des sœurs de Saint-Joseph au milieu du XVII ${ }^{\mathrm{e}}$ siècle a engendré une postérité considérable; encore aujourd'hui, la quarantaine de congrégations qui en sont issues comprend 20000 religieuses dans 56 pays. Un examen extrêmement minutieux des conditions dans lesquelles une telle initiative émerge durant la première moitié du XVIIe éclaire la dynamique religieuse de la période ; cette innovation institutionnelle marquée par l'œuvre de François de Sales combine l'héritage de la mystique rhéno-flamande avec l'influence décisive d'un jésuite. La ligne de ce dernier, le père Médaille, ne se comprendrait pas sans la conjoncture de la «nouvelle spiritualité jésuite » mise à jour pour cette période par Michel de Certeau.

Comme on le voit, le travail présenté par M.-L.G. est particulièrement stimulant quand on y trouve à la fois une différenciation très fine des courants spirituels et un repérage précis de la fécondation croisée qui engendre leurs postérités dans des parturitions qui sont loin d'être sans douleurs. À un moment où l'expérience mystique dut se faire très discrète, «l'autre est la médiation nécessaire qui rend possible l'action et la parole. La relation apostolique seule [...] permet d'articuler l'expérience mystique » (Michel de Certeau). M.-L.G. ajoute: "Pour le père Médaille, ce sont les femmes, les illettrés, le pauvre peuple des campagnes qui l'appellent hors du cercle où la mystique a été asséchée » (p. 507). Ce brassage des divergences aboutit à une certaine harmonie, que l'A. nous donne à voir en analysant deux tableaux peints par Georges de la Tour : «Le soleil des Rhénans chasse le noir de la désespérance humaine, le monde angélique se fait féminin et enfantin et fréquente la «Trinité créée », et le zèle ignatien se marie à la douceur salésienne dans la gamme des tons or et rouge brun » (p. 527).

Jacques Maître.

124.63

HOLDEN (Andrew).

Jehovah's Witnesses. Portrait of a Contemporary Religious Movement. Londres-New York, Routledge, 2002. 206 p. (bibliogr., glossaire, index).

Dans son introduction, l'auteur annonce une étude ethnographique des Témoins de Jéhovah fondée sur une observation participante avouée et sur des entretiens, destinée à appréhender leur vision de la réalité. Toutefois, nous ne décelons pas vraiment une description fine des pratiques et des conduites des fidèles. En fait, l'ouvrage s'apparente plus à une étude sociologique puisque A.H. examine le jéhovisme à la lumière des théories de la modernité et qu'il reprend des questions qui parcourent les études sur les groupes religieux minoritaires comme celles des rapports entre les fidèles et la société ambiante, les causes de la conversion, les conditions sociales et économiques qui ont entouré la naissance de ce mouvement, les réactions à l'échec des prophéties, le contrôle social interne et la régulation des croyances. Finalement l'ouvrage se situe dans la lignée de la thèse de James Beckord, Trumpet of Prophecy: A sociological study of the Jehovah's witnesses (Oxford, Basic, Blackwell, 1975) sans présenter l'avantage d'être la première étude sociologique publiée sur ce mouvement. Nous ne le critiquerons pas sur ce point car il est bon que des études de sciences sociales rappelant les éléments essentiels du jéhovisme paraissent de temps à autre. En revanche, nous le critiquons sur plusieurs points. 